Revue musicale OICRM

\title{
La partition sonore et musicale de Dunkerque (C. Nolan, 2017). Une expérience sensorielle inédite
}

\section{Emmanuelle Bobée}

Volume 5, numéro 2, 2018

Création musicale et sonore dans les blockbusters de Remote Control

URI : https://id.erudit.org/iderudit/1054150ar

DOI : https://doi.org/10.7202/1054150ar

Aller au sommaire du numéro

\section{Éditeur(s)}

Observatoire interdisciplinaire de création et recherche en musique (OICRM)

ISSN

2368-7061 (numérique)

Découvrir la revue

Citer cet article

Bobée, E. (2018). La partition sonore et musicale de Dunkerque (C. Nolan, 2017).

Une expérience sensorielle inédite. Revue musicale OICRM, 5(2), 125-148.

https://doi.org/10.7202/1054150ar
Résumé de l'article

Supervisée conjointement par Richard King et Hans Zimmer, la partition sonore et musicale de Dunkerque sous-tend l'élaboration d'un récit complexe, articulé autour de trois unités spatiotemporelles distinctes : une semaine sur la jetée, une journée en mer, une heure dans les airs. Les événements qui se déroulent au sein de chaque espace narratif obéissent à leur propre chronologie ; cependant, au fil de la narration, des croisements s'opèrent entre les personnages et des correspondances de situations s'établissent. L'action se décline au présent - un présent ubiquitaire, sans cesse renouvelé -, dans une temporalité dilatée par la charge émotionnelle et sensorielle véhiculée par la bande-son.

Nous montrerons que la conception sonore et musicale de Dunkerque répond à une volonté manifeste de conjuguer réalisme historique et spectacle immersif, dans une approche résolument novatrice et expérimentale. Outre le fait que la puissance sonore semble croître indéfiniment au gré des péripéties rencontrées par le jeune soldat anglais, la force et l'originalité de cette composition hybride résident dans la complémentarité et l'imbrication d'éléments constitutifs a priori disparates, tels que le chromatisme, la pulsation donnée par le tic-tac d'une montre, le sinistre gémissement des bateaux engloutis par la mer, ou encore les premières mesures de la variation « Nimrod » d'Edward Elgar. 


\title{
La partition sonore et musicale de Dunkerque (C. Nolan, 2017) : une expérience sensorielle inédite
}

\author{
Emmanuelle Bobée
}

\begin{abstract}
Résumé
Supervisée conjointement par Richard King et Hans Zimmer, la partition sonore et musicale de Dunkerque sous-tend l'élaboration d'un récit complexe, articulé autour de trois unités spatiotemporelles distinctes : une semaine sur la jetée, une journée en mer, une heure dans les airs. Les événements qui se déroulent au sein de chaque espace narratif obéissent à leur propre chronologie ; cependant, au fil de la narration, des croisements s'opèrent entre les personnages et des correspondances de situations s'établissent. L'action se décline au présent - un présent ubiquitaire, sans cesse renouvelé -, dans une temporalité dilatée par la charge émotionnelle et sensorielle véhiculée par la bande-son.

Nous montrerons que la conception sonore et musicale de Dunkerque répond à une volonté manifeste de conjuguer réalisme historique et spectacle immersif, dans une approche résolument novatrice et expérimentale. Outre le fait que la puissance sonore semble croître indéfiniment au gré des péripéties rencontrées par le jeune soldat anglais, la force et l'originalité de cette composition hybride résident dans la complémentarité et l'imbrication d'éléments constitutifs a priori disparates, tels que le chromatisme, la pulsation donnée par le tic-tac d'une montre, le sinistre gémissement des bateaux engloutis par la mer, ou encore les premières mesures de la variation «Nimrod » d'Edward Elgar.
\end{abstract}

Mots clés : Dunkerque ; Hans Zimmer ; Richard King ; bande-son ; partition musicale ; traitement sonore.

\begin{abstract}
Supervised jointly by Richard King and Hans Zimmer, the sound and musical score of Dunkirk underlies the elaboration of a complex narrative, articulated around three distinct spatiotemporal units: one week on the mole, one day at sea, one hour in the air. The events that occur within each narrative space follow their own chronology; however, throughout the narration, crossings take place between the characters, and correspondences of situations are established. The action comes in the present - a ubiquitous present, constantly renewed - in a temporality dilated by the emotional and sensory load conveyed by the soundtrack.

We will show that Dunkirk's sound and musical design responds to a clear desire to combine historical realism and immersive spectacle, in a resolutely innovative and experimental approach. Besides the fact that the sound power seems to grow
\end{abstract}


indefinitely according to the events met by the young English soldier, the strength and originality of this hybrid composition lie in the complementarity and the interweaving of a priori disparate components, such as the chromaticism, the pulsation given by the ticking of a watch, the sinister groan of boats swallowed by the sea, or the first measures of Edward Elgar's "Nimrod" variation.

Keywords: Dunkirk; Hans Zimmer; Richard King; soundtrack; musical score; sound treatment.

Sorti en juillet 2017, le dixième long métrage de Christopher Nolan retrace l'évacuation, en mai 1940, de plus de trois cent mille soldats alliés, encerclés par l'armée allemande dans la poche de Dunkerque - connue sous le nom de code "opération Dynamo ». Cette colossale reconstitution historique filmée au format IMAX, dont le budget a été estimé à cent millions de dollars, a été conçue à la fois comme un spectacle immersif et comme une épopée intime et subjective, qui dépeint les événements à travers le regard croisé des différents protagonistes (soldats, officiers, aviateurs, civils). L'objectif du réalisateur était aussi de parvenir à une fusion inédite entre l'image, les effets sonores et la musique (Guerrasio 2017). Afin de mener à bien ce projet ambitieux, il s'est entouré de deux fidèles collaborateurs habitués à travailler ensemble, déjà présents au générique de ses quatre précédents opus ${ }^{1}:$ le compositeur Hans Zimmer et le sound designer Richard King, qui a été récompensé à trois reprises par l'Oscar du meilleur montage son (notamment pour The Dark Knight : le chevalier noir et Inception) avant de partager sa quatrième statuette avec Alex Gibson pour la bande-son de Dunkerque.

La partition sonore et musicale du film sous-tend l'élaboration d'un récit complexe, articulé autour de trois unités spatiotemporelles distinctes : une semaine sur la jetée, une journée en mer, une heure dans les airs. Au sein de chaque espace narratif se déroulent des événements qui obéissent à leur propre chronologie, présentés en alternance dans un enchaînement de séquences de durées variables qui se succèdent presque toujours dans le même ordre. Cependant, au fil de la narration, des croisements s'opèrent entre les personnages, et des correspondances de situations s'établissent. Ainsi, l'un des deux aviateurs, contraint d'amerrir après avoir été touché par un avion ennemi, est repêché par deux héroïques civils anglais, Mr Dawson (Mark Rylance) et son fils Peter (Tom Glynn-Carney), qui naviguent en direction de Dunkerque sur un bateau de plaisance ; un peu plus tard, c'est le jeune soldat anglais (Fionn Whitehead) et l'un de ses compagnons d'infortune (Harry Styles) qui, après avoir été coincés sur la plage durant plusieurs jours et avoir réussi à prendre la mer à bord d'un chalutier abandonné, sont finalement hissés à bord du bateau de leurs compatriotes. Jalonné d'attaques aériennes et d'explosions assourdissantes, de naufrages spectaculaires et de combats aériens féroces entrecoupés de moments d'attente et de bouffées d'espoir, le récit converge vers la délivrance des militaires alliés, rapatriés en Angleterre par la

1 The Dark Knight : le chevalier noir (2008), Inception (2010), The Dark Knight Rises (2012) et Interstellar (2014). Auparavant, Hans Zimmer a composé la bande originale de Batman Begins (2005), en collaboration avec James Newton Howard, tandis que Richard King a supervisé les effets sonores dans Le Prestige (2006). 
flottille civile et sauvés in extremis d'un ultime bombardement grâce à l'intervention d'un pilote de la Royal Air Force.

Rares sont les moments de répit durant les cent-six minutes de projection de Dunkerque. Dans ce film de guerre aux allures de récit initiatique, la musique et les effets sonores, omniprésents dans la majorité des scènes, entretiennent une tension quasiment permanente jusqu'à l'ultime relâchement de l'épilogue. Au sein de ce continuum sonore et musical s'imbriquent quantités d'ambiances, de bruits, de battements sourds, d'ostinatos rythmiques, de nappes de synthétiseur et de lignes mélodiques originales ou empruntées. La force et l'originalité de cette composition hybride résident dans la complémentarité de tous ces éléments qui cohabitent, se croisent et se répondent dans un entrelacement constant. Si le travail minutieux mené par Richard King et son équipe autour de l'élaboration du sound design s'inscrit prioritairement dans une quête de la vraisemblance afin de procurer au spectateur des sensations intenses, la partition musicale de Hans Zimmer joue davantage sur le registre de la psychologie et de l'affect en exacerbant la tension émotionnelle du récit par l'intégration de sonorités bruitistes (notamment le «tic-tac » d'une montre absente de l'image) et l'utilisation récurrente du chromatisme, ainsi qu'en revisitant la célèbre variation « Nimrod » des Variations Enigma op. 36 d'Edward Elgar. Nous montrerons que la conception sonore et musicale de Dunkerque répond à une volonté de conjuguer "réalisme historique » et spectacle immersif, dans une approche résolument novatrice et expérimentale qui s'avère pleinement compatible avec ce genre de film destiné à une large audience.

\section{UN SOUND DESIGN EN QUÊTE DE RÉALISME}

Souhaitant se rapprocher au plus près de la réalité historique, Nolan tenait à ce que les effets sonores soient aussi réalistes que possible (Desowitz 2017). Lors de la phase de pré-production, Richard King a donc consulté de nombreux ouvrages historiques sur les événements qui se sont déroulés à Dunkerque pendant la Seconde Guerre mondiale, se penchant avec intérêt sur les témoignages de personnes qui avaient vécu cette période et en avaient conservé des souvenirs sonores. Ces témoignages portaient notamment sur le bruit caractéristique de sirène hurlante émis par certains bombardiers allemands, appelés Stuka - véritable arme psychologique conçue pour terroriser la population et décourager la fuite des militaires, le dispositif était constitué d'une sirène mécanique actionnée par une hélice, fixée au sommet de chaque train d'atterrissage ${ }^{2}$. Le sound designer rapporte les propos d'un soldat, selon lesquels

2 Dans Apocalypse Now (1979), Francis Ford Coppola met en scène un procédé similaire employé par l'armée américaine durant la guerre du Viêt-Nam, dans une perspective de guerre psychologique, consistant à diffuser une musique impressionnante (en l'occurrence, la «Chevauchée des Walkyries » de Richard Wagner) à un volume élevé, grâce à des haut-parleurs installés sur les hélicoptères. Cette technique, mise au point par les Opérations Psychologiques (PsyOps) de l'armée américaine, a effectivement été utilisée afin de terrifier la population et de démoraliser les troupes vietcongs. Selon Juliette Volcler et Victor A. Stoichita, « les cassettes audio diffusées depuis les airs participaient aussi à l'affirmation d'une toute-puissance technologique, destinée à établir un rapport de domination sur l'ennemi » (Volcler et Stoichita 2017). 
" c'était le son le plus infernal et le plus terrifiant qui se puisse rencontrer " (Desowitz 2017). Comme il n'existe plus d'avion de ce type en état de fonctionnement, il a dû recréer ce bruit en s'inspirant des enregistrements de l'époque :

We went out into a nearby desert with some real air sirens, which we over-cranked to make them more and more piercing - and to add some analog distorsion. To this more "pure" version of the sound we added an interesting assortment of other disparate sounds (King, cité dans Lambert 2017).

L'effet est particulièrement saisissant lors de la première scène située sur la plage, dans laquelle les soldats, qui attendent paisiblement de pouvoir embarquer, apparaissent soudain complètement terrifiés à l'approche des avions ennemis, s'égaillant en tous sens avant de se coucher au sol ou essayant vainement d'estimer la trajectoire des bombes (extrait vidéo 1). Le son strident des sirènes qui progresse irrépressiblement vers le registre aigu, couplé au ronflement des moteurs des trois bombardiers, tend à éveiller une incontrôlable sensation de danger imminent - un danger qui se concrétise au moment des explosions, treize impacts successifs dans l'axe de la caméra, de plus en plus proches du jeune soldat anglais qui apparaît au premier plan, couché à terre, sur la moitié gauche de l'écran. Le point de vue situé au ras du sol favorise l'immersion du spectateur au sein de l'action et l'incite à partager l'expérience du protagoniste. Le réalisme de la scène est renforcé par le traitement sonore fondé sur une corrélation étroite entre le point de vue et le point d'écoute : à mesure que les impacts se rapprochent, l'intensité sonore augmente, la bande de fréquences perceptibles s'élargit dans les médiums et le décalage lié à la vitesse de propagation du son se réduit progressivement jusqu'à ce que le son et l'image soient parfaitement synchronisés.

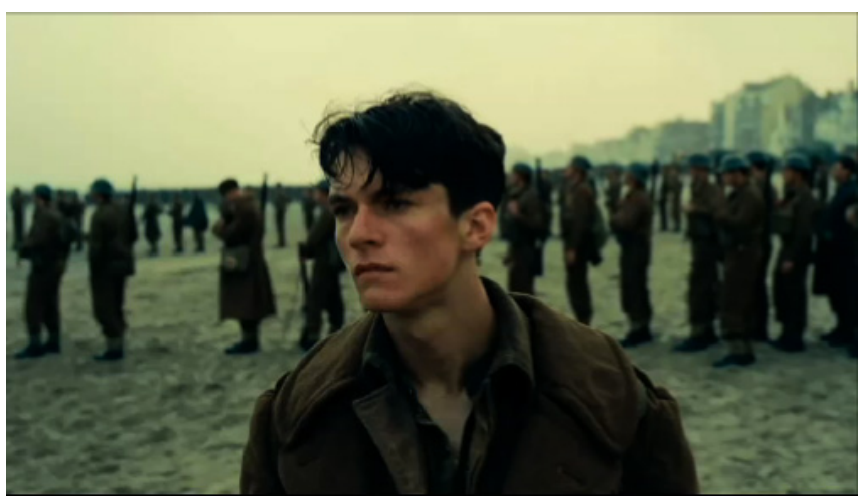

Extrait vidéo 1: Christopher Nolan, Dunkerque, bombardement de la plage par l'aviation allemande, 00:06:21-00:07:15 (C) Warner Bros.

King et son équipe ont réalisé de nombreux enregistrements en vol des différents modèles d'avions utilisés durant le tournage (bombardiers et chasseurs allemands, ainsi que les fameux Spitfire de l'armée britannique), plaçant jusqu'à vingt-quatre

3 "It was the most hellish, terrific noise you could ever encounter. " Toutes les traductions de l'anglais au français sont les nôtres. 
micros sur le fuselage, à proximité du moteur et de l'échappement ainsi qu'à l'intérieur du cockpit, et poussant le souci de réalisme au point de capter les vibrations de la carlingue afin de rendre perceptible à l'écran la sensation physique éprouvée par l'aviateur. Cette "symphonie de bruits de ferraille et de vibrations en perpétuel changement ${ }^{4}$ (Grobar 2017), qui rythme les séquences aériennes, plus denses, plus courtes et comportant davantage d'action, s'efface parfois au profit de la musique - notamment lorsque le pilote Farrier (Tom Hardy) décide hérö̈quement de retourner au combat alors que son réservoir est à sec - ou pour laisser place au dialogue entre les pilotes.

La conception du paysage sonore pour les séquences maritimes a également nécessité une quantité importante d'enregistrements de moteurs de bateaux, possédant des caractéristiques et des sonorités diverses. Le bruit récurrent du moteur du Moonstone (le bateau de plaisance piloté par Mr Dawson), qui rythme les séquences en mer, a fait l'objet d'une attention particulière : tandis que la plupart des enregistrements ont été effectués en Europe, celui-ci a été réalisé en Californie, dans le port de Marina del Rey (Lambert 2017). Selon des informations concordantes fournies par Zimmer et King (Ryzik 2017 et Desowitz 2017), il aurait été échantillonné et utilisé par le compositeur en tant que cellule rythmique dans la bande originale du film (il pourrait s'agir de la plage intitulée "Home » dans la bande originale du film, rythmée par un son itératif qui évoque une rotation mécanique). Le son des vagues frappant contre la coque, celui du vent soufflant sur le pont, les grincements provoqués par le roulis ainsi que tous les bruits liés à la navigation ont été captés pour pouvoir recréer de façon tangible la sensation éprouvée à bord d'un bateau. La dimension tragique et effrayante de certaines scènes, montrant d'imposantes embarcations chargées de soldats en train de chavirer après avoir été touchées par une bombe ou une torpille, repose en grande partie sur la puissance évocatrice de la bande-son. Outre les paquets d'eau qui se déversent à l'intérieur de la coque, comme par exemple lors de la scène du torpillage (00:38:32-00:41:01), la reconstitution sonore de l'événement nécessite de concevoir un agencement de bruits susceptible de rendre compte de la puissance des forces de torsion qui s'exercent sur le navire au moment du naufrage : "Imaginez un bateau en train de couler ", commente King. "La coque du bateau se déchire, grince et gémit, comme un gigantesque animal à l'agonie » (cité dans Grobar 2017).

Enfin, n'oublions pas que Dunkerque est un film de guerre, dans lequel les explosions, les tirs des mitrailleuses, les coups de fusil et de canon anti-aérien accompagnent les moments les plus intenses du récit. Chaque pièce d'armement possède une sonorité spécifique, que les ingénieurs du son John P. Fasal et Eric Potter se sont appliqués à restituer avec la plus grande précision. Le son de la mitrailleuse du Spitfire, que le commandant Bolton (Kenneth Branagh) semble reconnaître instantanément tandis que Farrier décoche une ultime rafale sur le bombardier allemand, a dû être recréé en raison d'un problème de surchauffe des appareils existants, la cadence de tir des mitrailleuses ne pouvant être maintenue à un niveau suffisant qu'en altitude.

\footnotetext{
4 "Symphony of rattles and vibrations, constantly changing".

5 "Imagine a ship sinking. [...] The hull of the ship itself is rending and creaking and groaning, like a huge animal dying. »
} 
En matière de technicité, de précision et d'inventivité, l'élaboration de la bande-son de Dunkerque s'apparente ainsi à un véritable tour de force. Le vaste panel de sonorités enregistrées ou recomposées à partir de témoignages, d'archives et de documents historiques contribue à donner la sensation d'un spectacle au réalisme saisissant, dans lequel chaque événement, chaque situation apparaît tangible et permet une immersion complète au sein de l'univers fictionnel. La puissance sonore de certains effets agit physiquement sur le spectateur dans le but d'annihiler toute velléité de résistance, de détachement ou de distanciation : "C'est une épopée et je voulais que le son soit épique ", déclare King. "Je voulais que les circonstances dans lesquelles se trouvent les personnages soient aussi terrifiantes et aussi passionnantes que possible ${ }^{6}$ (ibid.).

IMBRICATION DE LA MUSIQUE ET DES EFFETS SONORES

La bande-son de Dunkerque a été conçue de manière à ce que la musique et les effets sonores s'interpénètrent et se répondent dans un dialogue permanent. La volonté de fusionner la dimension sonore et la dimension musicale se traduit notamment par l'intégration, au sein de la partition créée par Hans Zimmer, de certaines sonorités bruitistes liées au contexte du récit : souffle intermittent et régulier évoquant le ressac de la marée ("The Mole ", "The Tide »), son de rotor ("Supermarine ", " Home »), bruits mécaniques et répétitifs ( Home»), pulsation sourde comme un battement de cœur ("The Mole », "Regimental Brothers »), etc. Renouant avec un procédé déjà employé dans Interstellar, Nolan a également demandé au compositeur de concevoir plusieurs pistes musicales à partir du son échantillonné d'une ancienne montre à remontoir, produisant un «tic-tac» caractéristique et très reconnaissable, afin d'inscrire concrètement l'idée de l'écoulement du temps au cœur du récit. Présent dès la séquence d'ouverture, cet élément sonore instaure d'emblée un sentiment d'inquiétude diffuse - on songe à la minuterie d'une bombe prête à exploser -, tandis qu'une poignée de soldats déambule dans les rues désertées de Dunkerque en attrapant au vol des tracts de l'armée allemande les intimant à se rendre. Des battements sourds, ainsi qu'une ligne mélodique extrêmement étirée, inspirée de la variation "Nimrod », jouée au synthétiseur avec une sonorité presque fantomatique, renforcent l'atmosphère d'accablement qui émane des lieux. Lorsqu'une fusillade éclate et que les soldats tombent l'un après l'autre sous le feu ennemi (extrait vidéo 2), le tic-tac toujours perceptible accompagne la fuite éperdue du seul survivant du groupe, exacerbant la tension émotionnelle de la scène, filmée caméra à l'épaule, et décuplant le sentiment d'urgence.

6 "It is an epic, and I wanted the sound to be epic. I wanted the circumstances that the characters find themselves in to be as terrifying and as exciting as possible. " 


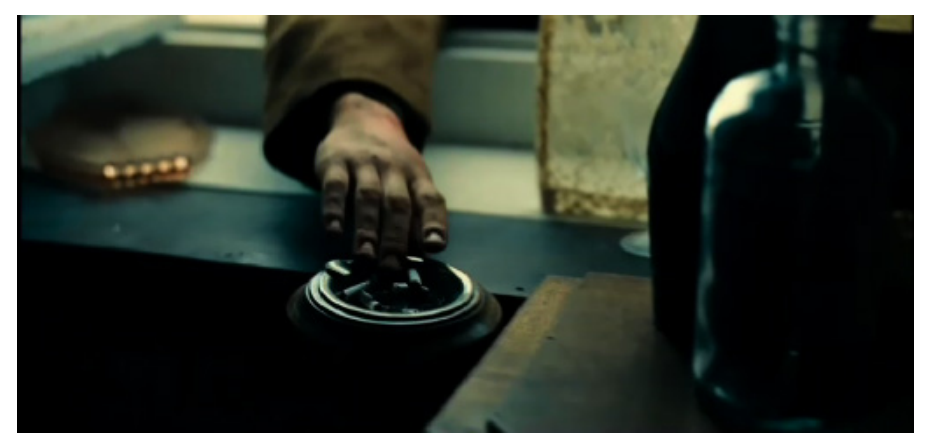

Extrait vidéo 2: Christopher Nolan, Dunkerque, fusillade et fuite dans les rues de Dunkerque, 00:01:43-00:02:34 (C) Warner Bros.

Le cliquetis du mécanisme de la montre réapparaît peu après au cours du premier plan sur les trois avions anglais qui survolent le channel, puis s'efface pour resurgir lors de certaines scènes particulièrement tendues (un combat aérien, la panique de l'aviateur prisonnier de son cockpit en pleine mer) ou dans des moments d'expectative et de suspense (l'interminable attente de la marée dans un bateau échoué pris pour cible par les Allemands, le survol de la plage par le Spitfire à court de carburant avant l'ultime attaque du bombardier allemand). Couplé à des nappes sonores dénuées de pulsation, il intervient comme un constant rappel du temps qui passe, et joue souvent en défaveur des protagonistes : à mesure que s'écoulent les minutes et les heures, le carburant s'épuise, le niveau de l'eau monte dans les espaces confinés et l'étau se resserre sur les soldats prisonniers de la plage de Dunkerque, sans cesse pilonnée par l'aviation ennemie. Simultanément, la pression croissante qui s'exerce sur les personnages est figurée par une pulsation sourde, parfois à la limite de l'audible, qui suggère un cœur en train de battre. Nolan confesse s'être inspiré du travail de John Carpenter, qui intègre fréquemment des pulsations cardiaques dans ses bandes originales (voir New York 1997, The Thing ou Le Village des damnés), pour imaginer une manière originale de combiner ces deux sonorités :

En coordination avec le département des effets sonores et de la musique, on s'est efforcés d'explorer la relation sonique entre un battement de cœur qui s'accélère et le « tic-tac » d'une montre. [...] On voulait créer une bande-son expérimentale autour de cette relation entre la représentation technologique du temps et sa forme organique, notre corps. Et comment cette relation change sous l'influence du suspense, de la peur ou d'une action intense (Nolan, cité dans Guedj 2017).

Tandis que l'allure du battement est soumise à d'incessantes fluctuations, accélérant et ralentissant en fonction des événements, le «tic-tac » de la montre reste immuable et constant. Du reste, sa pulsation mécanique et régulière, qui s'établit à 96 bpm, a servi de référence pour l'élaboration des différentes plages musicales utilisées au cours du film. La piste qui accompagne le générique de fin ("End Titles») est exécutée à ce tempo, tandis que "The Mole », "Shivering Soldier ", "We Need Our Army Back », " The Oil », " The Tide » et "Variation 15 » sont jouées deux fois plus lentement $(48 \mathrm{bpm})$. Des relations mathématiques simples ont également été appliquées pour définir des allures plus rapides, avec une augmentation d'un quart (soit 120 bpm) dans "Supermarine » et "Impulse » et d'un huitième (soit 108 bpm) dans « Regimental Brothers » et « Home». 


\begin{tabular}{|c|c|}
\hline «End Titles» & $96 \mathrm{bpm}$ \\
\hline $\begin{array}{l}\text { "The Mole» } \\
\text { "Shivering Soldier» } \\
\text { "We Need Our Army Back » } \\
\text { "The Oil» } \\
\text { «The Tide» } \\
\text { «Variation } 15 »\end{array}$ & $48 \mathrm{bpm}$, soit $96 / 2$ \\
\hline $\begin{array}{l}\text { « Supermarine » } \\
\text { «Impulse » }\end{array}$ & $120 \mathrm{bpm}$, soit $96^{*} 1,25$ \\
\hline $\begin{array}{l}\text { « Regimental Brothers » } \\
\text { « Home » }\end{array}$ & $108 \mathrm{bpm}$, soit $96 * 1,125$ \\
\hline
\end{tabular}

Tableau 1 : Tempi des plages musicales utilisées dans Dunkerque.

La relation mathématique qui s'établit entre les différents tempi des musiques $\mathrm{du}$ film n'est guère surprenante dans la mesure où Zimmer avoue volontiers un penchant pour les opérations et les jeux mathématiques appliqués à la composition (Cassidy 2010). Il est peu probable que l'oreille de l'audio-spectateur « moyen » soit assez fine pour repérer ces subtils rapports de tempi, mais on peut supposer qu'une telle démarche résulte de la volonté d'accroître la portée symbolique de la sonorité de la montre - qui ponctue le récit de façon récurrente - pour en faire un élément central de la conception de la bande musicale. Ce geste compositionnel témoigne, de surcroît, de l'importance de la représentation sonore du temps qui, de toute évidence, est une donnée essentielle dans la conception du récit filmique de Dunkerque.

\section{RÉCURRENCE DU CHROMATISME DANS LES THÈMES MUSICAUX}

La partition musicale composée par Hans Zimmer est caractérisée par une omniprésence de l'intervalle de demi-ton, qui contribue à instaurer un climat de tension permanente. Dès la quatrième minute du film, une nappe de cordes synthétiques, qui oscille en glissando sur le chromatisme la-sol\# dans le registre médium-aigu, apparaît lorsque le jeune soldat anglais aperçoit un autre militaire en train de recouvrir de sable le corps d'un camarade mort (à partir de 00:04:21). Couplée au tic-tac et aux battements décrits dans le paragraphe précédent, la nappe gagne peu à peu en intensité et se superpose à l'entrée d'un thème construit sur une gamme "ton/demi-ton " ("Regimental Brothers»), tandis que le jeune homme découvre les longues colonnes de soldats qui attendent d'être évacués vers l'Angleterre. À l'issue du bombardement de la plage par l'aviation allemande, un nouveau motif chromatique (mi-fa-mi) se déploie sur un rythme plus rapide, assurant la transition avec la séquence suivante qui montre l'effervescence des préparatifs sur le Moonstone, le bateau de plaisance de Mr Dawson, dans un petit port situé de l'autre côté de la Manche.

De retour sur la plage après une incursion dans les airs parmi les pilotes de la Royal Air Force, le récit se focalise sur le jeune soldat anglais et son compagnon, qui se fraient un chemin vers un bateau prêt à partir, en transportant au pas de course un 
blessé sur un brancard (extrait vidéo 3). La scène est sous-tendue par une succession de notes piquées aux cordes, enchaînées sur un tempo rapide, qui oscillent entre le si bémol 3 et le la 3 en passant par des hauteurs intermédiaires sur une échelle micro-intervallique, créant un effet de suspense et d'agitation amplifié par la prise de vue saccadée et les mouvements de caméra qui accompagnent le déplacement des personnages. Cet ostinato mélodico-rythmique se propage ensuite dans les deux autres espaces narratifs, ponctué de façon intermittente par des nappes de synthétiseur qui soulignent le caractère dramatique de l'action. Un peu plus loin dans le récit, une nappe oscillant sur le même demi-ton dans le registre aigu, à la manière d'une sirène, s'avère le prélude à une nouvelle attaque aérienne particulièrement dévastatrice. Durant la première heure du film, les occurrences de chromatismes répétés ponctuent régulièrement la narration sans que l'on y prête forcément attention, instaurant une atmosphère inquiétante, voire anxiogène, qui maintient le spectateur dans un état d'alerte permanent.

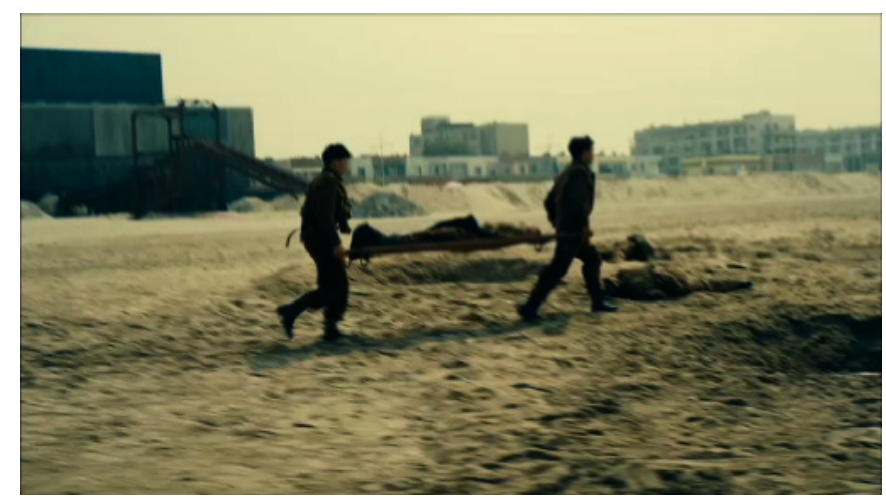

Extrait vidéo 3 : Christopher Nolan, Dunkerque, transport d'un blessé au pas de course sur la plage de Dunkerque, 00:10:11-00:12:14 C Warner Bros.

Cette profusion de sonorités oppressantes qui tapissent les images du film se double d'une utilisation récurrente de l'intervalle de demi-ton dans la majorité des plages musicales composées par Zimmer - à l'exception de celles qui sont inspirées de la variation "Nimrod ». Ainsi, la ligne mélodique de la piste intitulée "Shivering Soldier » est constituée d'une succession de demi-tons séparés par des intervalles de tierce mineure dans un mouvement descendant :

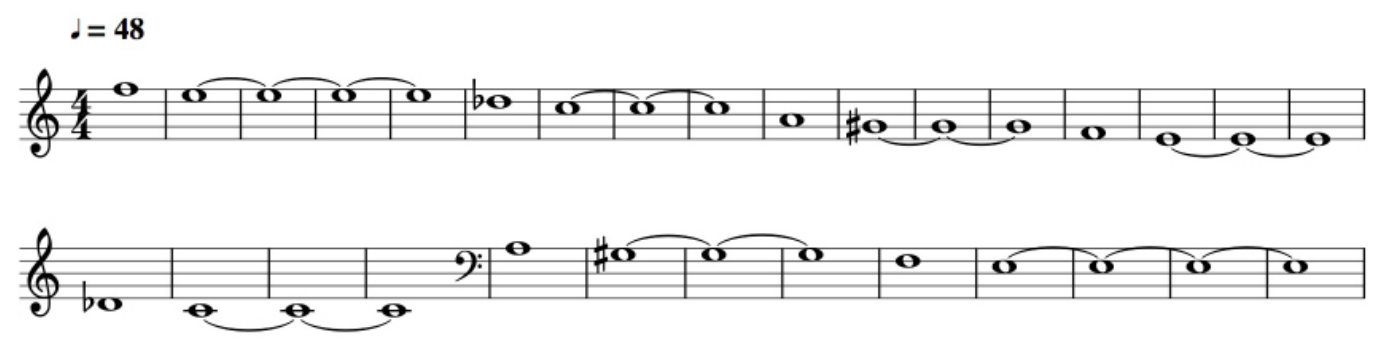

Exemple 1: Hans Zimmer, Dunkerque, cue "Shivering Soldier», ligne mélodique.

Transcription personnelle à partir de la bande originale (00:20-02:50).

La mélodie de "We Need Our Army Back» est sensiblement identique, à ceci près qu'elle est exécutée à l'octave supérieure. Ces deux thèmes, utilisés lors des 
scènes avec le soldat britannique (Cillian Murphy) repêché en mer par Mr Dawson et son fils, puis au cours d'une longue séquence retraçant le pathétique retour au pays des soldats évacués par la flottille britannique, donnent à entendre une lente et inexorable chute qui s'opère par paliers successifs et semble ne jamais devoir s'arrêter, dans un contexte harmonique instable qui abolit toute notion du temps. Illustrant la débâcle des forces alliées et des ravages psychologiques de la guerre, ils véhiculent un sentiment de tristesse et d'abattement mêlé d'une certaine étrangeté, que renforce l'utilisation d'un timbre synthétique dans la séquence du débarquement des soldats. Dans la version qui accompagne la première conversation entre le propriétaire du bateau et le militaire sauvé des eaux, la sonorité aiguisée des cordes et l'exécution des chromatismes en glissando laisse de surcroît planer une impression de menace diffuse, comme un sombre présage des tragiques événements à venir - au cours d'une altercation avec Mr Dawson, le soldat, terrifié à l'idée de retourner vers la plage de Dunkerque, cause involontairement la mort de George (Barry Keoghan) en voulant contraindre son sauveur à faire demi-tour.

Le thème intitulé "Home " est bâti sur une gamme par tons ascendante dont chaque note se résout sur son demi-ton descendant :

\lrcorner$=108$

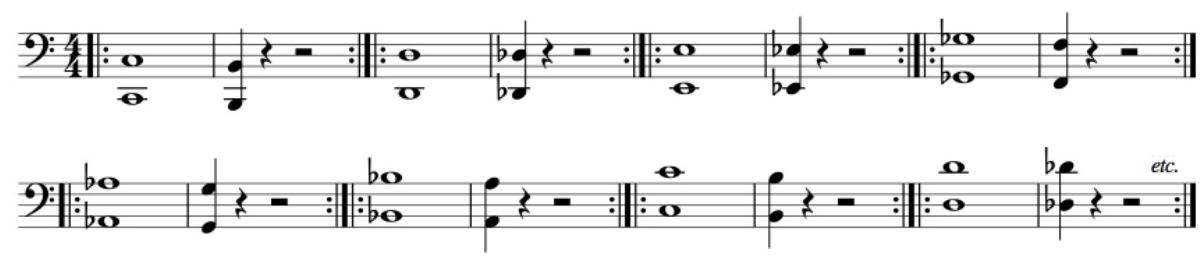

Exemple 2 : Hans Zimmer, Dunkerque, cue "Home», ligne mélodique. Transcription personnelle à partir de la bande originale (00:00-01:11).

Nous reviendrons plus loin sur les caractéristiques de cette piste musicale, élaborée selon le principe de la gamme de Shepard afin de produire l'illusion auditive d'un mouvement ascendant ininterrompu. Le choix du timbre utilisé, sorte de rugissement synthétique rythmé par des bruits mécaniques, de même que la dynamique qui anime chacun des motifs successifs, donnent à cette composition un caractère brutal, sévère et implacable. Ici, l'effet recherché est l'instauration d'une tension croissante (amplifiée dans le film par l'accélération du tempo), la figuration d'une catastrophe en marche que rien ne peut arrêter, dont la dimension inexorable s'incarne dans le mouvement ascendant infini. Mêlé aux bruits de moteurs et au son des explosions, ce thème tonitruant se déploie sur une succession de plans aériens (01:10:32-01:11:26) montrant successivement le bombardement d'un destroyer allié par l'aviation allemande et le chavirage de l'imposante embarcation, qui bascule sur le flanc en laissant échapper dans la mer une nappe de liquide sombre, comme un animal éventré.

Plus statique, mais aussi plus inquiétante, la ligne mélodique d' "Impulse » est fondée, comme la première partie de «Supermarine ", sur une alternance de demi-tons descendants et ascendants autour de la tonique la dans les registres médium et grave : 


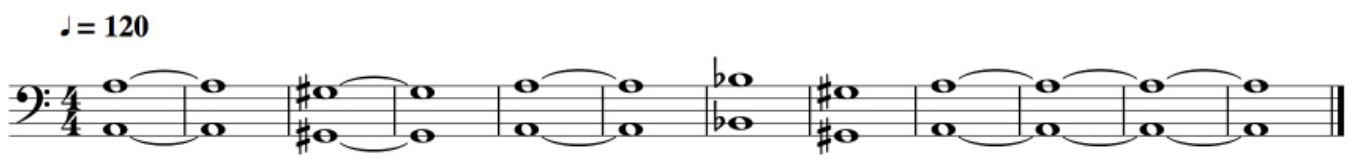

Exemple 3 : Hans Zimmer, Dunkerque, cue «Impulse ", ligne mélodique.

Transcription personnelle à partir de la bande originale (01:23-01:47).

Un son continu oscillant entre le si et le do de la troisième octave ajoute une tension supplémentaire à cette plage musicale, qui intervient lors d'un combat aérien au cours duquel Farrier abat un chasseur allemand, avant d'apercevoir dans son rétroviseur un bombardier ennemi - précisément celui qui pilonnera le destroyer quelques instants plus tard. Soutenue par une rythmique plus présente, cette mélodie apparaît également dans la première partie de "Supermarine ", dont une version alternative, condensée et comportant quelques ajouts de parties instrumentales qui renforcent le caractère pulsé du morceau, accompagne une autre séquence de combat aérien, particulièrement longue et mouvementée. Dans la piste qui figure sur le $\mathrm{CD}$ de la bande originale, elle mène à une phrase musicale construite sur la gamme «ton/demi-ton » ascendante, en partant de la note la, énoncée au début du film sur un tempo plus lent :

$J=120$

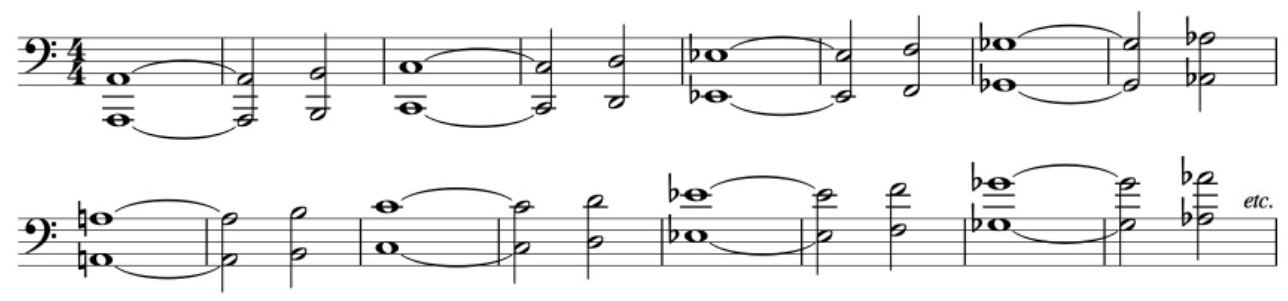

Exemple 4: Hans Zimmer, Dunkerque, cue "Supermarine», utilisation de la gamme «ton/demi-ton». Transcription personnelle à partir de la bande originale (06:52-07:24).

Cette phrase constitue aussi le matériau mélodique principal de « The Oil », une composition à la structure complexe dans laquelle des diminutions rythmiques successives du motif musical se superposent progressivement à l'élément initial ${ }^{7}$, produisant des dissonances et des décalages rythmiques qui entretiennent la confusion et donnent finalement l'impression d'un indescriptible chaos. La piste musicale, dont nous avons effectué une transcription simplifiée de la portion la plus représentative (extrait audio 1), a de surcroît subi des modifications lors de son intégration au récit filmique : la pulsation est irrégulière et le rythme y apparaît plus déstructuré, en raison notamment d'un décalage entre les différentes voix et l'ostinato de la partie supérieure.

7 On assiste ici à un effet de tintinnabuli comme dans le Cantus in memoriam Benjamin Britten d'Arvo Pärt : la même ligne mélodique est déclinée à des vitesses (rythmiques) différentes, en valeurs brèves à l'aigu et en valeurs longues dans le grave. 

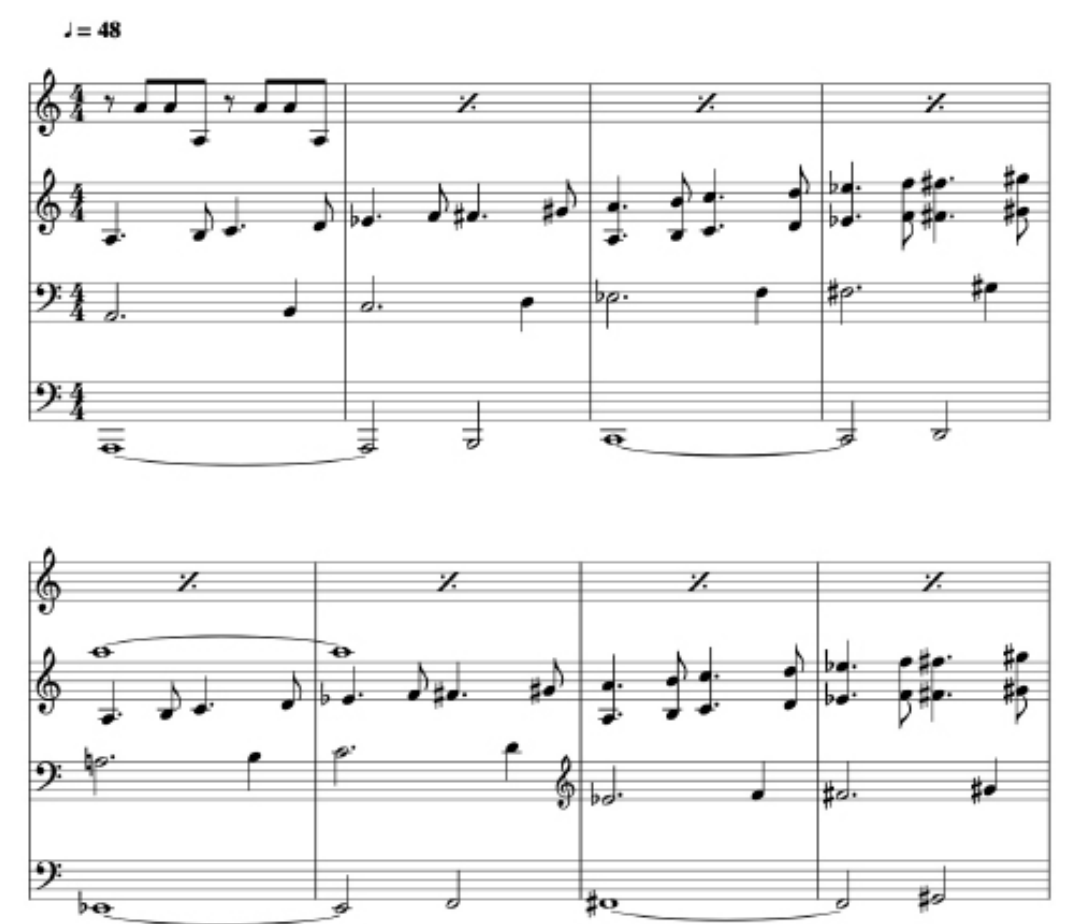

Exemple 5 : Hans Zimmer, Dunkerque, cue "The Oil». Transcription simplifiée à partir de la bande originale (03:40-04:20).

Extrait audio 1: Hans Zimmer, Dunkerque, cue "The Oil», 03:40-04:20 @ Sony Music Entertainment. Écouter.

À travers ce recensement détaillé, mais non exhaustif, il apparaît clairement que le chromatisme est un élément constitutif majeur de la partition musicale de Dunkerque. Décliné dans sa forme élémentaire (motifs, ostinatos) ou intégré au sein d'une véritable structure mélodique, il participe activement à l'instauration d'une atmosphère de tension, d'excitation et de suspense que le compositeur, conformément au cahier des charges imposé par Nolan, s'est employé à façonner dans la perspective d'une intensification graduelle. Toutefois, la pression se relâche par intermittence pour laisser place au sentiment, via les nombreux emprunts à la variation dite "Nimrod " qui émaillent la bande musicale du film.

\section{AutOUR DE LA VARIATION « NimRoD »}

Dédicacée par le compositeur et chef d'orchestre britannique Edward Elgar (1857-1934) à son meilleur ami, un éditeur de musique allemand nommé August Jaeger, la neuvième des Variations Enigma est sans doute l'une des œuvres postromantiques les plus populaires au Royaume-Uni. Inspirée par le deuxième mouvement de la Sonate pour piano $n^{\circ} 8$ en do mineur de Ludwig van Beethoven, plus connue sous le nom de "Sonate pathétique ", elle doit son appellation à un personnage de l'Ancien Testament, Nimrod, décrit dans la Genèse comme un " puissant chasseur devant l'Éternel ». Par cette référence, il semble qu'Elgar ait voulu remercier son ami pour ses conseils et son soutien dans les moments de doute en lui conférant une stature de héros biblique, le terme allemand pour désigner un chasseur étant « jäger ». 
Créées à Londres en juin 1899, sous la direction de Hans Richter, les Variations Enigma ont recueilli un succès critique et public immédiat, apportant au compositeur la reconnaissance de son talent et une notoriété internationale. L'œuvre dans son ensemble reste aujourd'hui l'une des plus jouées de son catalogue, avec la première marche de Pomp and Circumstance ; toutefois, la variation "Nimrod » a connu un retentissement particulier dès lors qu'elle a été intégrée, en 1930, au programme musical joué lors des cérémonies officielles du Remembrance Sunday. Son exécution, chaque deuxième dimanche de novembre, dans le cadre de cet hommage rendu aux soldats et civils britanniques ayant servi la patrie durant les deux guerres mondiales et lors des conflits ultérieurs, a infléchi sa signification première en l'investissant d'un sentiment patriotique et en lui conférant le statut de symbole de la grandeur du peuple prêt à se sacrifier pour son pays : " Par un processus irréversible d'association, "Nimrod" a acquis une vie indépendante en tant qu'élégie nationale ${ }^{8}$ (Rushton 1999, p. 46).

Né d'un père britannique et d'une mère américaine, Christopher Nolan a passé une partie de son enfance en Angleterre et possède la double nationalité. C'est probablement l'une des raisons qui explique son attachement à cette pièce, qu'il trouve "insupportablement émouvante $"{ }^{9}$ (cité dans Ryzik 2017) et dont il a suggéré l'utilisation en tant qu'élément thématique du film - l'autre raison fait écho à son histoire personnelle, la variation "Nimrod " ayant été diffusée lors des obsèques de son père (ibid.). Plusieurs arrangements ont été réalisés par Zimmer, qui n'a utilisé tout au plus que les quatre premières mesures du thème, prenant le parti de ralentir considérablement le mouvement métronomique du morceau et d'étirer à l'extrême chacune des notes de la mélodie.

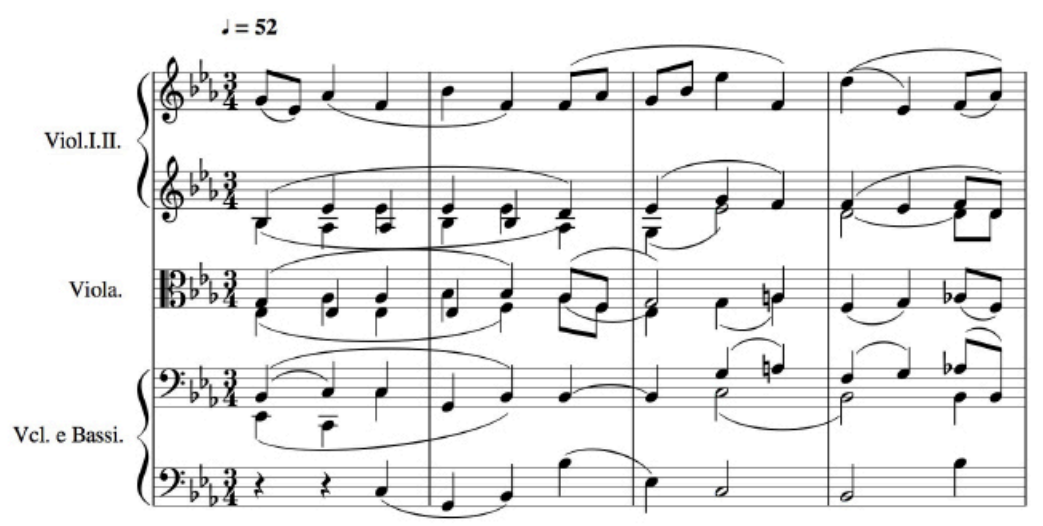

Exemple 6 : Edward Elgar, Variations Enigma, "Variation IX (Nimrod)", mes. 1-4.

Une version relativement proche de l'original ("Home ", à partir de 04:04) apparaît dans le récit au moment de l'arrivée de la flottille civile anglaise, qui est accueillie triomphalement par les soldats (extrait vidéo 4). La dimension épique de cette séquence, qui intervient après plusieurs scènes mouvementées et oppressantes rythmées par une composition très anxiogène, est renforcée par la référence à cette musique chargée d'histoire. La sensation d' " harmonie » et d'apaisement véhiculée

8 "Through irreversible processes of association, "Nimrod" has acquired an independent life as a national elegy. "

9 "Unbearably moving. " 
par cette pièce ancrée dans le langage tonal est d'autant plus prégnante que la majeure partie de la bande musicale est fondée sur une succession de dissonances dont la résolution est sans cesse différée. La tonalité ( $m i$ bémol majeur), la ligne mélodique et les harmonies restent inchangées par rapport à la partition de référence ; seul le tempo est substantiellement modifié, avec une exécution sept fois plus lente que la version initiale - environ 7,2 bpm au lieu de $52 \mathrm{bpm}$-, qui accentue le caractère solennel et majestueux de la pièce tout en permettant aux images de se déployer dans la durée. On peut également noter l'ajout d'une partie rythmique aux cordes, qui ne figure pas dans la partition d'origine, ainsi que l'intégration de sonorités synthétiques (notamment un battement régulier dans le registre grave), répondant vraisemblablement à un désir d'actualiser le morceau et d'en proposer une version plus conforme aux attentes du public dans le contexte d'un blockbuster. En effet, la satisfaction de la quête du plaisir musical requiert la mise en place de procédés d'écriture relativement stéréotypés, tels que l'intégration de percussions et d'ostinatos dans les scènes d'action, tout en préservant l'équilibre entre nouveauté et prédictibilité (Guadalupe Silveira 2013, p. 32 et suivantes).

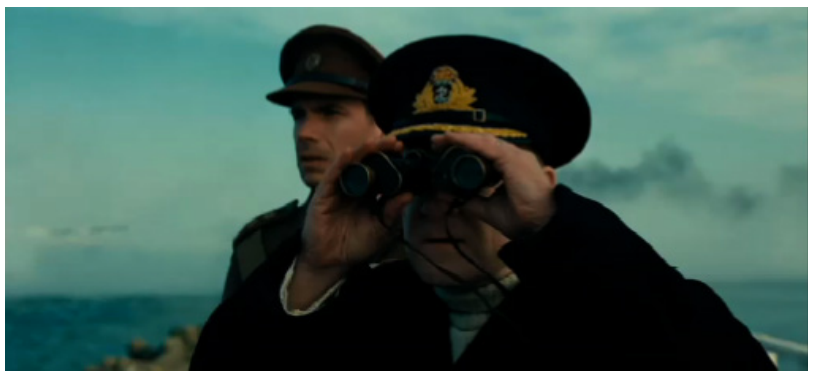

Extrait vidéo 4: Christopher Nolan, Dunkerque, arrivée de la flottille civile anglaise, 01:13:28-01:15:27 (c) Warner Bros.

Une autre version, intitulée « Variation 15 »- en référence aux quatorze variations présentes dans la partition d'Elgar -, s'appuie tout d'abord sur les deux premières mesures du thème, avant de proposer un développement original qui accompagne la séquence finale durant presque six minutes. La trame harmonique est plus statique et la pulsation extrêmement flottante, donnant la sensation d'une mélodie dilatée à l'extrême. Bien que Zimmer ait conservé le mode majeur, il émane de ce thème une profonde mélancolie, un sentiment de tristesse empreint de solennité et de retenue, qui s'accorde parfaitement avec l'atmosphère du retour des soldats vaincus, soulagés de rentrer au pays mais honteux d'avoir été réduits à la fuite devant le camp adverse. Le caractère de la pièce évolue à mesure que le jeune soldat anglais lit à haute voix un article paru dans la presse nationale, qui relativise la déroute du camp allié et redonne espoir en la victoire en encourageant coûte que coûte la poursuite du combat ${ }^{10}$. Aux deux tiers du morceau, le sentiment d'accablement se mue peu à peu en un sentiment héroïque et patriotique, tandis que la musique se fait plus rythmée et que le tempo accélère

10 Ce texte est un assemblage d'extraits du discours prononcé par Winston Churchill devant la Chambre des Communes le 4 juin 1940. 
légèrement, accompagnant les images de l'aviateur qui, dans un geste de rébellion, met le feu à son appareil avant de se rendre à l'ennemi.

Cette longue plage musicale constitue l'aboutissement d'une série de références plus ou moins explicites à la variation "Nimrod ", disséminées au cours du récit. Dans "The Mole », la piste musicale utilisée pendant l'ouverture du film, on reconnaît la courbe mélodique du thème original, transposée un ton et demi plus bas et exécutée sur un tempo lent (48 bpm) au synthétiseur avec un son de cordes synthétiques caractérisé par une longue durée d'attaque et de relâchement ("release»), qui lui donne un caractère presque fantomatique. Précisons que la valeur rythmique de chacune des notes de la mélodie a été multipliée par six - une croche devient une blanche pointée -, ce qui aboutit à une vitesse d'exécution sensiblement identique à celle des deux versions décrites plus haut.

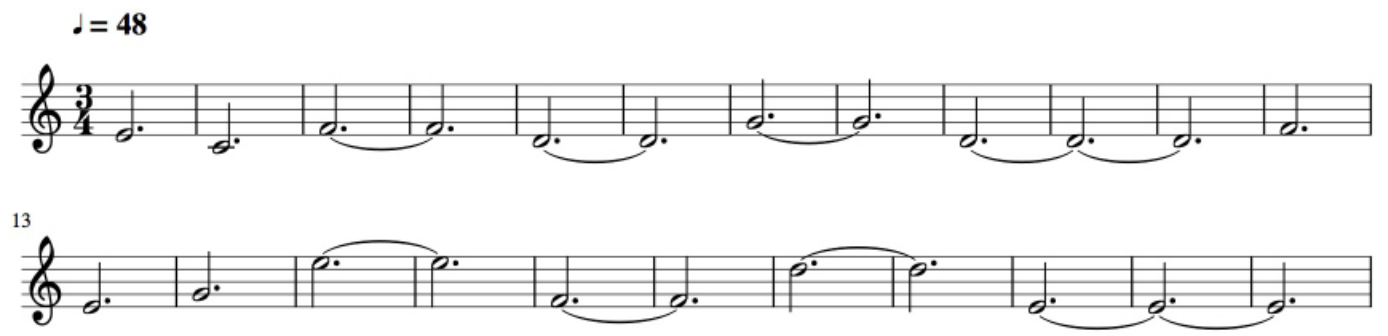

Exemple 7 : Hans Zimmer, Dunkerque, cue "The Mole», ligne mélodique. Transcription personnelle à partir de la bande originale (00:30-01:56).

La ligne mélodique a été modifiée dans le troisième tiers (mes. 15-23 dans l'exemple 7 ci-dessus), de sorte que le saut de quarte initial se transforme en saut de sixte, accentuant la polarisation de la mélodie autour de la note mi (qui ouvre et referme le thème) et renforçant du même coup l'impression générale de statisme. Si l'on distingue des hauteurs dans le registre médium, les appuis harmoniques sont difficilement identifiables et les valeurs rythmiques ne peuvent être quantifiées précisément ; dans l'ensemble, il émane de cette adaptation une impression de flottement qui rend plus tangible encore la solitude et l'errance des soldats dans les rues désertes de Dunkerque. Plus loin, lorsque les trois officiers anglais discutent sur la jetée et s'accordent sur la situation désespérée de la poche de Dunkerque en évoquant la perspective de pertes humaines considérables, une mélodie sensiblement identique à celle de "The Mole » se profile sous les dialogues (00:20:16-00:21:30). Derrière la froideur et l'objectivité du discours militaire, cette occurrence musicale dessine en filigrane le portrait d'hommes sensibles et compatissants, tout en glissant une allusion aux soldats morts pour la patrie, comme un hommage funèbre anticipé.

De courtes citations apparaissent ensuite dans des moments particulièrement dramatiques ou critiques, par exemple quand le bateau de la Croix Rouge commence à couler près de la jetée sous le regard effaré du commandant (00:28:05-00:28:27), lors de l'amerrissage de Collins (00:42:43-00:43:30) ou lorsque le soldat français est démasqué par ses compagnons (01:07:54-01:08:30). Si le contexte harmonique de ces occurrences est ambigu, la citation des trois premières notes du thème qui ponctue le moment décisif au cours duquel Farrier fait demi-tour pour revenir combattre l'aviation allemande, bien que sa réserve de carburant soit presque épuisée, se superpose à une progression harmonique en mode majeur (comme dans la version 
d'origine), qui se greffe sur des images relativement neutres - la mer en plan incliné subjectif, alternant avec des gros plans sur le visage impassible du pilote en train d'effectuer la manœuvre - en leur apportant une touche de grandeur et d'héroïsme.

Enfin, le retour des soldats sur la plage aux premières lueurs de l'aube, après le torpillage du destroyer à bord duquel ils avaient réussi à embarquer, s'accompagne d'une version particulièrement sombre et cafardeuse, intitulée « The Tide ». Émaillée d'un son pulsé qui évoque le ressac de la marée, la mélodie, exécutée aux cordes dans les registres grave et extrême aigu, se déploie sur une pédale de la, qui suggère un contexte harmonique mineur, accentuant le sentiment de désolation qui émane des images. Rythmées par cette musique plaintive, les visions funestes des soldats qui tentent vainement de mettre à l'eau des embarcations repoussées par le courant, d'un homme désespéré qui se jette à la mer et des cadavres ramenés par la marée se succèdent à l'écran, dans une esthétique visuelle relativement dénuée d'affect et caractérisée par une température de couleur froide, allant du bleu au gris (extrait vidéo 5).

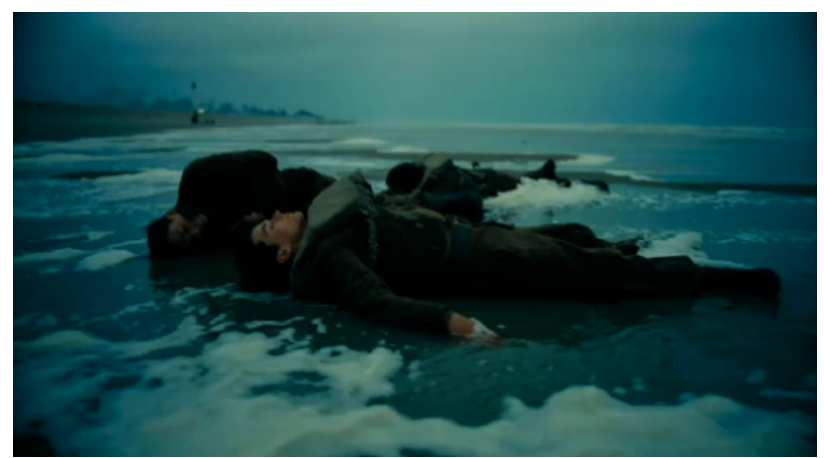

Extrait vidéo 5 : Christopher Nolan, Dunkerque, visions funestes sur la plage de Dunkerque, 00:49:22-00:50:58 (c) Warner Bros.

Ces occurrences musicales inspirées par la variation « Nimrod» véhiculent tour à tour un sentiment d'espoir, d'inquiétude ou de profonde tristesse. Le contexte harmonique, les transformations du matériau mélodique, l'ajout d'éléments rythmiques, le choix des timbres, l'orchestration et la vitesse d'exécution sont autant de paramètres qui modifient la perception du thème. Il est intéressant de noter qu'au-delà de cette ambivalence émotionnelle, imputable en partie au caractère multivoque de la ligne mélodique et en partie au travail de façonnage effectué par le compositeur, chacune des plages musicales exalte à sa manière le sentiment patriotique. Dans les moments de doute comme dans les moments d'espérance, elles visent à susciter une profonde empathie vis-à-vis des soldats qui accomplissent leur devoir ou tentent simplement de survivre.

\section{LA BANDE-SON, FACTEUR DE CONTINUITÉ}

Comme nous l'avons rappelé dans l'introduction, les trois unités spatiotemporelles juxtaposées dans le récit sont relativement étanches - excepté lors des points de jonction - et l'action qui s'y déroule ne présente aucun caractère de simultanéité. Le réalisateur a fait le choix de définir trois espaces narratifs indépendants, qui se succèdent en alternance à l'écran, afin de livrer au spectateur une vision plurielle et subjective des événements, vécus de l'intérieur par les protagonistes qui observent 
la même réalité sous des angles différents. La multiplicité des points de vue produit un récit fragmenté, caractérisé par de constants changements de décor et par une absence de synchronisme ou de continuité temporelle entre les scènes situées sur la plage, en mer et dans l'espace aérien. Toutefois, la bande-son a été conçue de manière à atténuer cette impression de morcellement du récit et à instaurer une forme de continuité entre les différents espaces narratifs, reliés par des ponts sonores ou par des plages musicales qui englobent plusieurs segments. Ainsi, par exemple, la portion du film comprise entre 00:10:11 et 00:15:58, qui parcourt deux fois le cycle terre-mer-air, est ponctuée par une plage musicale ininterrompue qui débute par des notes répétées sèchement aux cordes sur un intervalle de demi-ton et s'enrichit peu à peu, dans un crescendo dramatique, de nappes de synthétiseur dans les registres aigu et médium, puis dans le registre grave, avant de s'interrompre brutalement au passage d'un avion ennemi au-dessus de l'un des Spitfire. Cette pièce au caractère très oppressant exacerbe le sentiment d'urgence initié par la course des deux soldats vers le bateau en partance et contribue à propager au sein des deux autres espaces narratifs l'atmosphère de tension et d'agitation inhérente à l'action qui se déroule sur la plage de Dunkerque.

Plus subtile, la technique du pont sonore consiste à désynchroniser légèrement les entrées et sorties des plages sonores ou musicales par rapport à la délimitation visuelle des scènes, de sorte que le son se prolonge au début de la séquence ou du plan suivant, ou commence légèrement en avance, sur les dernières images de la séquence ou du plan précédent. Elle est employée de façon quasiment systématique durant les soixante-dix premières minutes du film, s'appuyant la plupart du temps sur le prolongement ou l'anticipation de plages musicales (souvent des nappes de synthétiseur ou des parties rythmiques) dont l'intensité varie en fonction du contexte. En l'absence de musique, c'est une pulsation sourde relayée par les vibrations d'un moteur ou par une boucle rythmique sur un tempo identique qui fait office de pont sonore entre deux scènes, dans un continuum qui souligne l'importance fondamentale du rythme, véritable « dimension-pivot ${ }^{11}$ de la bande-son.

$\mathrm{Au}$ fil du récit, la frontière entre les trois espaces narratifs devient de plus en plus poreuse : la route des personnages se croise et le récit met en scène des situations similaires qui se succèdent dans un montage alterné - l'eau monte dans le cockpit de Collins et dans le chalutier investi par les Highlanders, des soldats sont hissés dans l'embarcation des Dawson et sur les bateaux civils à proximité de la plage, etc. Dès l'instant où Collins embarque à bord du bateau des deux Anglais, ce ne sont plus seulement des ponts sonores qui relient les scènes, mais une véritable continuité musicale. La piste intitulée "Home », utilisée lors de la scène du bombardement et de l'impressionnant chavirage du destroyer, se poursuit sans interruption dans les séquences suivantes, qui montrent successivement une discussion entre Collins et Peter Dawson autour de George - dont l'état se dégrade et n'inspire que peu d'espoir au pilote -, puis le départ tardif d'un bateau sur la jetée, et enfin l'affolement des soldats

11 Selon Michel Chion, la « dimension-pivot » se définit comme un « aspect commun appartenant à des éléments sonores considérés comme différents de nature (parole, musique, bruit), et permettant soit de passer progressivement de l'un à l'autre, soit de les faire ressembler l'un à l'autre »(Chion 2010, p. 418). 
réfugiés dans un chalutier qui dérive vers le large en prenant l'eau de toutes parts. La musique tisse un lien entre ces différentes situations et souligne ce qu'elles ont en commun : l'idée d'une catastrophe en marche à laquelle les personnages ne peuvent qu'assister, impuissants, réduits à l'état de spectateurs passifs. Le thème initial se fond ensuite avec un arrangement de la variation "Nimrod "sur lequel s'effectue une jonction entre la terre et la mer - les premiers bateaux de la flottille civile arrivent sur la plage et l'évacuation des soldats s'organise. À l'issue de cette séquence qui introduit la première véritable lueur d'espoir dans le récit, une nouvelle plage musicale fait son entrée, "The Oil ", qui relie une quinzaine de segments durant plus de neuf minutes consécutives (01:15:46-01:25:00). Cette séquence extrêmement tendue, articulée autour du sauvetage par les Dawson des soldats rescapés du naufrage et de l'intervention de Farrier qui parvient à neutraliser le bombardier ennemi, matérialise le point de rencontre des trois unités narratives. Le récit converge vers ce moment particulièrement intense, au cours duquel les protagonistes se retrouvent au cœur de la même situation, réunis par le hasard ; la structure de la piste musicale, conçue autour de l'entrelacement de trois lignes mélodiques construites sur un motif identique, plus ou moins dilaté ou condensé, fait d'ailleurs écho à cette conjonction qui précède le dénouement. La conclusion du récit est également placée sous le signe de la continuité musicale, avec une nappe prolongée de synthétiseur sur la note mi, relayée par trois thèmes successifs - dont la « Variation 15 » qui accompagne la séquence de l'épilogue -, s'enchaînant de manière fluide, sans que les entrées et sorties n'attirent particulièrement l'oreille du spectateur. Ce parti pris découle vraisemblablement d'une volonté de neutraliser l'effet de distanciation qui pourrait résulter de la narration non linéaire. L'impression de continuité générée par le montage de la musique et des effets sonores permet de préserver la faculté du spectateur à s'immerger dans le récit de ces histoires croisées, sans que son attention ne soit détournée de l'action par la soudaine prise de conscience d'assister à une représentation fictive.

\section{LA GAMME DE SHEPARD : UNE IDÉE DE L'ÉTERNITÉ}

Parmi les procédés acoustiques utilisés dans l'élaboration de la partition de Dunkerque, Zimmer s'est inspiré d'une illusion auditive connue sous le nom de "gamme de Shepard", afin d'instaurer une tension croissante et de donner la sensation d'un mouvement ascendant qui semble ne jamais devoir s'arrêter (Ryzik 2017). Mis au point en 1964 par Roger N. Shepard, un scientifique américain spécialisé dans les sciences cognitives, ce procédé s'appuie sur la synthèse préalable par ordinateur des "Shepard tones ", des sons périodiques complexes qui résultent de l'addition de signaux sinusö̈daux de même intensité, séparés par des intervalles d'octave. Cet empilement d'octaves " a pour effet de rendre la tessiture de chaque son ambiguë ", puisqu' " il est difficile d'estimer à quelle octave se situe la note " (Féron 2010, p. 159). Lorsque plusieurs sons se succèdent pour former les degrés d'une gamme ascendante (ou descendante) et que celle-ci est répétée à plusieurs reprises, on obtient l'illusion d'un mouvement perpétuellement ascendant (ou descendant). Cet artifice a été utilisé notamment dans le jeu vidéo Super Mario 64, développé par Nintendo dans les années 1990, pour accompagner l'ascension d'un escalier sans fin par la célèbre mascotte de la société japonaise (extrait vidéo 6). 


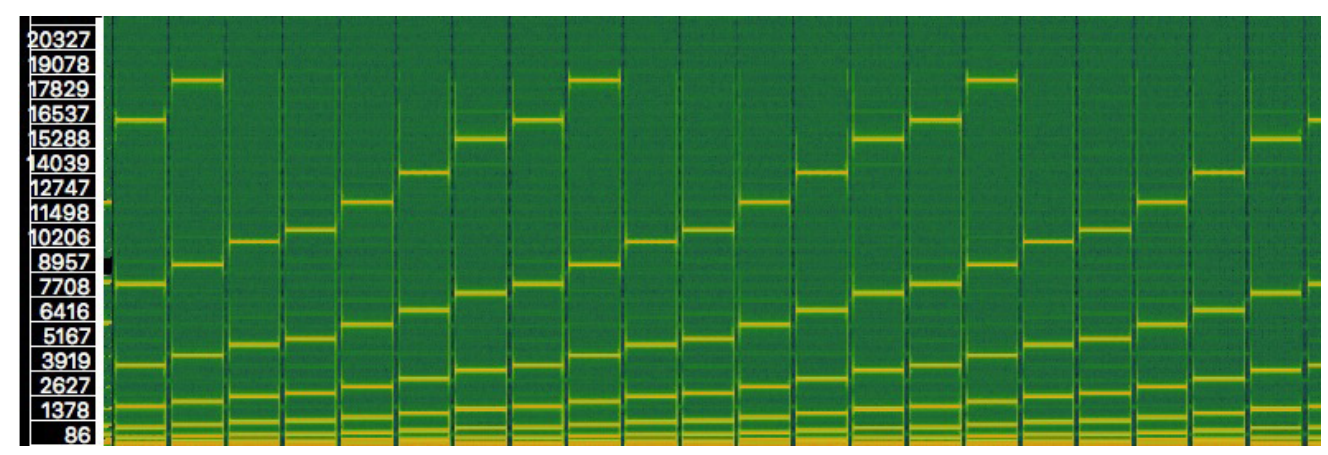

Exemple 8: Sonagramme d'une gamme de Shepard (échelle des fréquences linéaires).

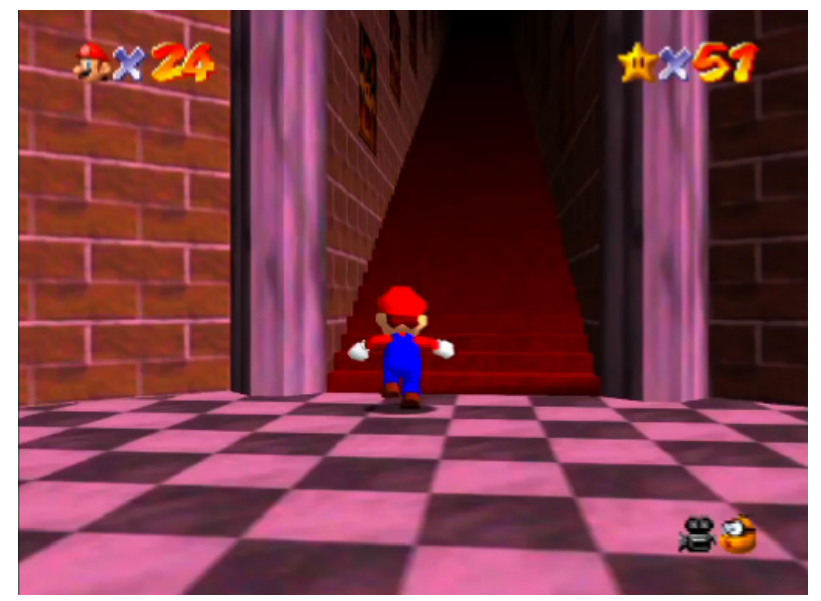

Extrait vidéo 6 : Shigeru Miyamoto, Super Mario 64, ascension d'un escalier infini (c) Nintendo.

\section{David Wessel et Jean-Claude Risset soulignent que}

ce comportement paradoxal évoque l'escalier montant (ou descendant) indéfiniment de Roger [sic] Penrose ; [...] la montée indéfinie donne l'illusion que la fréquence du son augmente sans cesse, ce qui ne peut bien sûr être le cas sans que le son sorte du domaine audible» (Wessel et Risset s.d.).

La référence à cet objet impossible, qui met en évidence la relativité de la perception humaine et traduit " un conflit entre les perspectives locales et la compréhension globale ${ }^{12}$ (Hartmann 1998, p. 147), nous renvoie directement au cinéma de Nolan et, en particulier, à une séquence d'Inception dans laquelle Arthur explique à la jeune Ariane les possibilités infinies de l'architecture paradoxale conçue par un esprit en train de rêver (Blu-ray, de 00:39:54 à 00:40:25). L'intérêt du réalisateur pour le domaine de l'illusion n'est pas récent ; en effet, il s'est manifesté auparavant dans Le Prestige, récit articulé autour de la rivalité entre deux prestidigitateurs, dont la bande originale, composée par David Julyan, incluait déjà des parties synthétiques élaborées selon la méthode de Shepard:

Pour la musique du Prestige, j'ai utilisé des sons électroniques pour produire des effets que je ne pouvais pas créer avec un orchestre... Il y en a beaucoup dans certains 
morceaux comme Colorado Springs, où l'on entend une gamme de Shepard en fond. [...] C'est un très bel effet que nous avons choisi ensemble, Chris et moi. Il m'a permis de créer des fonds très texturés pour les sons de l'orchestre (Julyan, cité dans Bordwell et Thompson 2014, p. 470).

Lors de l'écriture du scénario de Dunkerque, Nolan explique qu'il s'est inspiré de l'effet de mouvement perpétuel ascendant produit par cette illusion psychoacoustique pour concevoir l'entrelacement des trois chronologies narratives, dans la perspective d'une intensification constante du récit (Guerrasio 2017). À sa demande, Zimmer s'est également appuyé sur ce principe pour accroître l'intensité de certaines plages musicales qui accompagnent des moments paroxystiques. Ainsi, la dernière partie de "Supermarine » (CD, piste 4, à partir de 06:53), qui sous-tend un combat aérien mouvementé au cours duquel les deux aviateurs anglais abattent un chasseur ennemi avant de parvenir à neutraliser un bombardier au-dessus de la Manche, s'appuie sur une succession de gammes ton/demi-ton ascendantes superposées à des intervalles d'octaves et réitérées à quatre reprises pendant environ une minute. Le sonagramme de cet extrait musical fait apparaître clairement la répétition du signal acoustique, qui présente un profil similaire à celui de la gamme de Shepard, à ceci près que l'on observe une intensification progressive dans le registre aigu - le compositeur a renforcé l'effet de mouvement ascendant en ménageant une entrée progressive des pistes musicales situées dans la partie haute du spectre sonore.

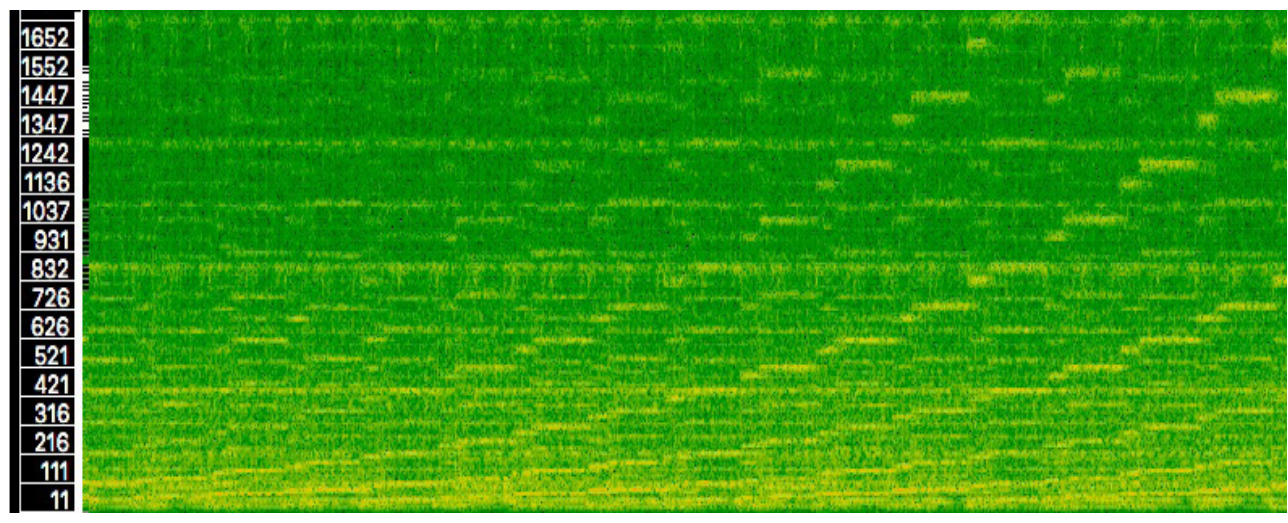

Exemple 9: Sonagramme du cue « Supermarine » de 06:53 à 07:56 (échelle des fréquences linéaires).

Le segment final de "The Oil » (CD, piste 9, à partir de 05:00), qui repose sur une construction rigoureusement identique, apparait comme le point culminant de la pièce, dont il apaise soudainement les divergences rythmiques pour installer une atmosphère d'excitation, fondée sur la répétition et l'amplification progressive de la ligne mélodique principale. Il apparaît au cours d'une séquence mouvementée, qui fait suite au naufrage du destroyer à bord duquel le jeune soldat anglais et la troupe des Highlanders ont embarqué après avoir enfin réussi à quitter la côte à bord d'un chalutier abandonné. La scène débute par un combat aérien entre Farrier et le bombardier allemand responsable de la catastrophe, puis les plans s'enchaînent dans un rythme soutenu, filmés alternativement depuis l'espace aérien et depuis l'espace maritime - c'est-à-dire à bord du bateau de Mr Dawson, venu secourir les naufragés, et dans ses environs immédiats. Suscitant un mouvement de panique générale, le 
bombardier revient à la charge avant d'être abattu par le pilote anglais et de s'écraser au milieu d'une nappe de fuel qui s'enflamme immédiatement (extrait vidéo 7). Par son mouvement continuellement ascendant, la musique contribue efficacement à accroître la tension émotionnelle de la scène, créant un climat de suspense et d'anxiété renforcé par l'ajout de percussions et une accélération progressive du tempo - la version intégrée au film diffère légèrement de celle qui figure sur le CD.

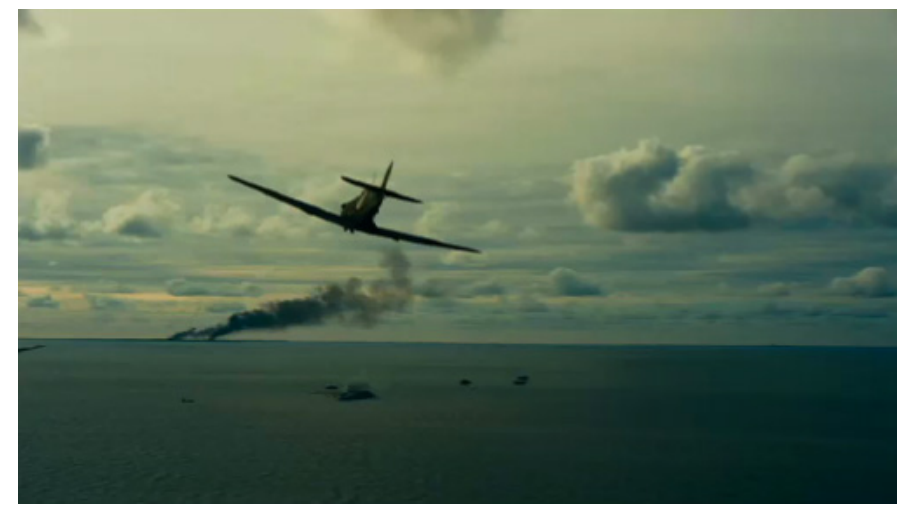

Extrait vidéo 7: Christopher Nolan, Dunkerque, combat aérien et sauvetage des rescapés du naufrage du destroyer, 01:23:51-01:25:00 @ Warner Bros.

Le même procédé est appliqué dans la première partie de la piste intitulée " Home » (extrait audio 2), dont la mélodie possède aussi un caractère globalement ascendant, qui semble poursuivre un mouvement perpétuel. Dans cette plage musicale au caractère bruitiste relativement affirmé, Zimmer a également introduit un son de rotor - nettement perceptible à partir de 00:20 - qui évolue en glissando vers le registre aigu, suivant la technique du "glissando de Shepard-Risset ", élaborée par le compositeur Jean-Claude Risset ${ }^{13}$ en 1967 :

Comme les sons de Shepard, ce son montant continu est obtenu en ajoutant plusieurs sons simples restant à intervalle d'octave, alors que leurs fréquences augmentent : lorsqu'un son atteint une fréquence assez élevée, son amplitude baisse graduellement jusqu'à devenir inaudible, cependant qu'une composante grave est rajoutée insidieusement pour renouveler le stock d'octaves, qui défilent ainsi sans cesse vers l'aigu (Wessel et Risset s.d.).

Extrait audio 2: Hans Zimmer, Dunkerque, cue "Home»,00:00-03:29 (C) Warner Bros. Écouter.

Dans la bande-son de Dunkerque, Zimmer recourt également à une illusion auditive appliquée à la dimension rythmique, afin de créer une sensation d'accélération perpétuelle. Cette technique, mise au point en 1970 par Kenneth Knowlton, repose sur la superposition et l'interaction de deux strates de pulsations dont la fréquence et l'amplitude sont contrôlées de façon indépendante, de sorte que certaines d'entre elles disparaissent progressivement $\mathrm{du}$ champ de perception à mesure que les pulsations se rapprochent (Féron 2010, p. 158-161). Elle est appliquée notamment sur un son

13 Utilisé dans sa pièce intitulée Mutations (1969), le "glissando de Shepard-Risset " a également été employé à la fin d'un morceau de Pink Floyd, Echoes (album Meddle, 1971). 
percussif intermittent de hauteur indéfinie, dont le timbre semble simultanément évoluer vers les fréquences aiguës. Le battement frénétique et précipité de cette boucle synthétique, possible figuration d'un cœur qui s'accélère et bat la chamade, exacerbe le sentiment d'urgence et d'appréhension qui émane des scènes qu'elle accompagne : l'évacuation du bateau touché par une torpille, l'amerrissage forcé de Collins (extrait vidéo 8 ) et enfin les deux ultimes attaques aériennes.

Extrait vidéo 8: Christopher Nolan, Dunkerque, amerrissage forcé de Collins, 01:01:39-01:02:13 (C) Warner Bros.

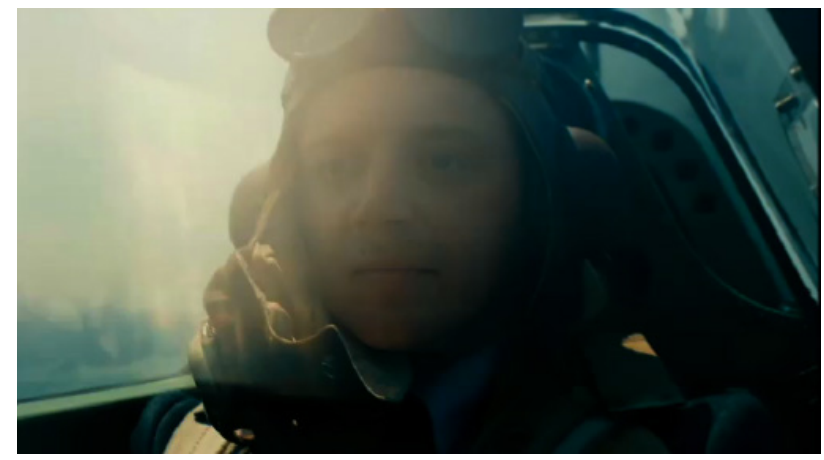

Les recherches et les tâtonnements qu'a nécessités, plusieurs mois durant, l'élaboration de la bande musicale s'expliquent par la difficulté à mettre en œuvre et à combiner ces procédés expérimentaux, rarement utilisés au cinéma. La stratégie compositionnelle initiée par Nolan et développée par Zimmer vise avant tout à produire des sensations nouvelles et inédites. Toutefois, au-delà de l'effet recherché en matière de puissance sonore et de spectaculaire, le compositeur explique que les illusions psychoacoustiques se réfèrent pour lui à une notion plus abstraite, qui fait écho à la construction narrative du film : "It's the idea of eternity and endlessness [...]. It's the idea of playing a trick on time itself " (Zimmer, cité dans Lau 2017). Désorienter le spectateur pour mieux capter son attention et le happer dans une fiction où se côtoient de multiples temporalités, telle semble être la règle de conduite adoptée par le duo dans ce récit déroutant qui, par bien des aspects, déroge aux canons du cinéma hollywoodien.

"Dunkerque est plutôt une expérience qu'un film » ${ }^{14}$, résume Hans Zimmer (ibid.). La recherche de sensations physiologiques générées par la bande-son y apparaît primordiale, au sens où elle conditionne l'adhésion du spectateur au monde fictionnel, représenté à travers la subjectivité des différents personnages qui traversent le récit. Le défi consistait à concevoir une œuvre cinématographique immersive et spectaculaire, visant un large public, tout en renouvelant la forme d'un genre maintes fois exploré : le film de guerre. La quête du réalisme sonore, qui nécessite un travail minutieux de restitution ou de recréation des bruits diégétiques, s'accompagne d'une volonté d'accroître l'intensité dramatique des scènes dans un mouvement perpétuellement ascendant, qui s'inspire des illusions visuelles et auditives de Lionel Penrose et Roger Shepard. La complémentarité de la musique et du sound design repose sur un 
équilibre des forces en présence qui, dans ce cas précis, ne s'impose pas comme une évidence et nécessite une approche particulière de la part du compositeur : " Il n'y a aucune chance que je puisse rivaliser avec le son d'une fusillade ou des vagues qui se brisent, alors je dois aborder cela d'un point de vue différent ${ }^{15}$, précise Zimmer (dans Ryzik 2017). La variété des procédés musicaux qui s'inscrivent dans cette recherche d'intensité permet un renouvellement permanent de la partition qui accompagne le film dans un crescendo cyclique, entrecoupé de respirations salvatrices. Elle témoigne d'un langage en perpétuelle évolution qui, certes, s'appuie sur des fondamentaux (richesse du timbre, rythmes appuyés, ostinatos, chromatisme) mais n'exclut pas une démarche expérimentale fondée sur la recherche d'une cohérence interne avec le scénario complexe du film. Parvenant à relier la forme et le fond, à insuffler de la simplicité dans la complexité - et vice versa -, la partition sonore et musicale de Dunkerque contribue à donner corps concrètement à cette fiction paradoxale, qui revisite un épisode de la grande Histoire dans une perspective intensément subjective.

\section{BIBLIOGRAPHIE}

Bordwell, David, et Kristin Thompson ([1999] 2014), L'art du film. Une introduction, traduit de l'américain par Cyril Béghin, Louvain-la-Neuve, De Boeck Supérieur.

Cassidy, Kevin (2010), "Q\&A. Composer Hans Zimmer », The Hollywood Reporter, https://www. hollywoodreporter.com/news/qampa-composer-hans-zimmer-25455, consulté le 12 avril 2018.

Chion, Michel ([1990] 2013), L'Audiovision. Son et image au cinéma, Paris, Armand Colin.

Chion, Michel ([2003] 2010), Un art sonore, le cinéma. Histoire, esthétique, poétique, Paris, Cahiers du cinéma.

Desowitz, Bill (2017), " "Dunkirk”. How Christopher Nolan Created Unique, Rhythmic, and Very Loud Sounds of War ", Indiewire, http://www.indiewire.com/2017/07/dunkirk-how-christophernolan-very-loud-sounds-of-war-1201860117/, consulté le 10 avril 2018.

Féron, François-Xavier (2010), «L'art du trompe-l'oreille rythmique », Intermédialités, n 16, p. 145-165.

Grobar, Matt (2017), «"Dunkirk" Sound Editor Richard King on Immersing an Audience in a Pivotal WWII Moment ", Deadline, http://deadline.com/2017/11/dunkirk-richard-king-sound-editoroscars-interview-1202210900/, consulté le 16 avril 2018.

Guadalupe Silveira, Carlos Henrique (2013), La musique de film. Introduction à l'étude des attentes musicofilmiques du spectateur, thèse de doctorat, Université Lumière Lyon 2.

Guedj, Philippe (2017), "Christopher Nolan. "Dunkerque est un appel à l'héroïsme collectif" ", Le Point Pop, http://www.lepoint.fr/pop-culture/cinema/christopher-nolan-dunkerque-est-un-appela-1-heroisme-collectif-20-07-2017-2144438 2923.php, consulté le 12 avril 2018.

Guerrasio, Jason (2017), "Christopher Nolan Explains the Biggest Challenges in Making His Latest Movie "Dunkirk" Into an "Intimate Epic" ", Business Insider, http://www.businessinsider.fr/us/ christopher-nolan-dunkirk-interview-2017-7, consulté le 10 avril 2018.

Hartmann, William M. (1998), Signals, Sound, and Sensation, New York, AIP-Press/Springer.

15 "There's no way I'm going to beat the sound of gunfire or crashing waves, so I have to approach it from a different point of view. " 
Lambert, Mel (2017), " Richard King Talks Sound Design for Dunkirk », PostPerspective, http://postperspective.com/richard-king-talks-dunkirks-sound-design/, consulté le 16 avril 2018.

Lau, Melody (2017), "Hans Zimmer Explains the Audio Trickery that made Dunkirk Audiences Nauseous ", $C B C$, http://www.cbc.ca/radio/q/blog/hans-zimmer-explains-the-audio-trickerythat-made-dunkirk-audiences-nauseous-1.4311684, consulté le 10 avril 2018.

Nolan, Christopher (2017), Dunkerque, 2 Blu-rays, Warner Bros., 1000638056.

Nolan, Christopher (2010), Inception, 2 Blu-rays, Warner Bros., 1000121977.

Rushton, Julian (1999), Elgar: Enigma Variations, Cambridge, Cambridge University Press.

Ryzik, Melena (2017), "Ticking Watch, Boat Engine, Slowness. The Secrets of the "Dunkirk" Score ", The New York Times, https://www.nytimes.com/2017/07/26/movies/the-secrets-of-the-dunkirkscore-christopher-nolan.html, consulté le 10 avril 2018.

Volcler, Juliette, et Victor A. Stoichita (2017), «Armes sonores et musiques d'ambiance», Terrain : revue d'ethnologie de l'Europe, $\mathrm{n}^{\circ} 68$ (octobre), p. 132-149, http://journals.openedition.org/terrain/16360, consulté le 10 août 2018 .

Wessel, David, et Jean-Claude Risset (s.d.), " Illusions auditives », Encyclopcedia Universalis, http://www.universalis.fr/encyclopedie/illusions-auditives/, consulté le 5 mai 2018.

Zimmer, Hans (2017), Dunkirk. Original Motion Picture Soundtrack, Sony Music Entertainment, 88985461482. 